\title{
The role of auxiliary oxidants in maintaining redox balance during phototrophic growth of Rhodobacter capsulatus on propionate or butyrate
}

\author{
David J. Richardson, Glenn F. King, David J. Kelly, Alastair G. McEwan, Stuart J. Ferguson, and J. Barry Jackson
} Arch Microbiol (1988) 150:131-137

Due to an unfortunate error, part of Table 2 and two sentences in this paper, were wrongly printed. Table 2 should have read as follows:

Table 2. The molar ratio of TMAO or DMSO reduced per carbon substrate assimilated during exponential phototrophic growth of $R b$. capsulatus strain AD2. The consumption of oxidant and carbon substrates were measured using ${ }^{1} \mathrm{H}-\mathrm{NMR}$ as described in the text. The succinate/TMAO and one of the malate/TMAO molar ratios were measured at $300 \mathrm{MHz}$. Samples were from cultures in exponential phase. n.d. $=$ not determined

\begin{tabular}{lll}
\hline $\begin{array}{l}\text { Carbon } \\
\text { source }\end{array}$ & $\begin{array}{l}\text { Ratio of oxidant reduced per carbon } \\
\text { source consumed }\end{array}$ \\
\cline { 2 - 3 } & TMAO & DMSO \\
\hline Propionate & 0.98 & 0.90 \\
Butyrate & 2.05 & 2.30 \\
Malate & 0.1 & n.d. \\
Succinate & 0.09 & n.d. \\
\hline
\end{tabular}

On page 136, line 6 from bottom (left) the text should read: (Table 1). Measurements of both $\mathrm{NO}_{2}^{-}$and TMA accumulation as well as consumption of DMSO in strains expressing the appropriate auxiliary pathways showed that the oxidants were extensively reduced during growth.

On page 136 , line 29 from bottom (right) the text should read:

It is significant that the molar ratio of TMAO or DMSO reduced per butyrate consumed is approximately 2.0 (Table 2). 